\title{
Philosophiques
}

\section{Le salut par les affects : La joie comme ressort du progrès éthique chez Spinoza}

\section{Syliane Charles}

Volume 29, numéro 1, printemps 2002

Spinoza sous le prisme de son anthropologie

URI : https://id.erudit.org/iderudit/009564ar

DOI : https://doi.org/10.7202/009564ar

Aller au sommaire du numéro

Éditeur(s)

Société de philosophie du Québec

ISSN

0316-2923 (imprimé)

1492-1391 (numérique)

Découvrir la revue

Citer cet article

Charles, S. (2002). Le salut par les affects : La joie comme ressort du progrès éthique chez Spinoza. Philosophiques, 29(1), 73-87.

https://doi.org/10.7202/009564ar d'utilisation que vous pouvez consulter en ligne.

https://apropos.erudit.org/fr/usagers/politique-dutilisation/ 


\title{
Le salut par les affects : La joie comme ressort du progrès éthique chez Spinoza
}

\author{
SYLIANE CHARLES \\ Université de Montréal \\ Sy_Charles@yahoo.ca
}

\begin{abstract}
RÉSUMÉ. - Cet article propose une relecture du cheminement éthique dans la connaissance chez Spinoza sous l'angle de l'affectivité qui l'accompagne. L'analyse du passage de l'imagination à la raison lui réserve donc une place privilégiée, et ce non pour justifier ce passage, qui se fait mécaniquement par la comparaison entre elles d'idées du premier genre, mais pour expliquer la naissance d'un désir de connaissance dès qu'est donnée ne serait-ce qu'une idée vraie. De plus, l'affectivité joyeuse est également présente dans la connaissance vraie, et nous esquissons les grandes lignes d'une interprétation faisant de l'intuition l'expérience affective des idées données par la connaissance rationnelle.
\end{abstract}

\begin{abstract}
This paper proposes a revisitation of the ethical progress in knowledge in Spinoza from the perspective of the affectivity that accompanies it. Consequently, joy deserves a privileged place in the analysis of the transition from imagination to reason, not as a means to explain this transition itselfwhich is done automatically through the comparison of ideas of the first kind with each other-but to give an account of the arising of a desire for knowledge as soon as be it just one true idea is given. Furthermore, the affect of joy is also present in true knowledge, and this paper provides a broad outline of an interpretation making of intuition the affective experience of the ideas provided by rational knowledge.
\end{abstract}

Le bien suprême est pour lui [l'homme] de parvenir à jouir — avec d'autres individus, si faire se peut - d'une telle nature [...]. [Cette nature supérieure] est la connaissance de l'union que l'esprit possède avec toute la Nature. Telle est donc la fin vers laquelle je tends, à savoir, acquérir une telle nature supérieure et travailler à ce que beaucoup d'autres l'acquièrent avec moi ${ }^{1}$.

L'éthique de Spinoza pourrait être qualifiée de philosophie de la jouissance, et s'il va de soi que Spinoza présente une conception progressive de la connaissance humaine, l'essentiel de son projet réside dans le fait que celle-ci est aussi, de manière immédiate, une conception progressive du bonheur humain : l'accomplissement éthique n'est pas un accomplissement de la connaissance seule, mais à travers celle-ci, de l'individu total. Or, il importe de voir que si la joie est la finalité de l'éthique, elle en est aussi le chemin. Nous voudrions

1. TIE 13-14, G II, 8 ; c'est nous qui soulignons. Les abréviations utilisées dans ce texte suivent la consigne établie dans les Studia Spinozana pour les citations, et nous n'indiquons que la référence dans l'édition Gebhardt ( $\mathrm{G}$ suivi du tome puis de la page). Pour le Traité de la réforme de l'entendement, nous suivons la traduction d'Alexandre Koyré (Paris, Vrin, 1994). Pour tous les autres ouvrages, nous utilisons l'édition des Euvres de Charles Appuhn (Paris, GFFlammarion, 4 vol., 1965). 
présenter ici une explication de la théorie de la connaissance qui montre combien l'affectivité fait partie intégrante du connaître. Une étude exhaustive de cette question nécessitant cependant beaucoup plus de place qu'il ne nous en est imparti ici, avec en particulier l'analyse des dimensions affectives et expérientielles du regard d'éternité, nous nous contenterons, dans le cadre de cet article, de souligner la présence de la joie à chaque degré du perfectionnement dans la connaissance, et de tracer les grandes lignes d'une interprétation du rôle de celle-ci dans la progression elle-même. Nous nous attacherons par conséquent avant tout à montrer la continuité d'un genre à l'autre, tout en indiquant à chaque point de transition le rôle possible de la joie dans l'explication de la dynamique progressive.

\section{Hiérarchie des genres de connaissance}

On trouve des exposés de la théorie de la connaissance de Spinoza, et plus précisément des différentes manières de connaître, dans les trois ouvrages éthiques de Spinoza : le Court Traité (KV 2/1), le Traité de la réforme de l'entendement (TIE 19-24) et l'Éthique (E 2P40S2). Malgré quelques fluctuations dans le nombre (trois ou quatre) et dans le nom de ces modes de connaissance, on s'accorde généralement pour reconnaître que Spinoza n'a pas considérablement varié dans sa conception, même si inversement celle-ci semble n'avoir jamais été parfaitement établie ${ }^{2}$. De ce fait, nous nous permettrons de nous référer ici simplement à la dernière expression de cette théorie du progrès dans la connaissance, celle de l'Éthique, qui est la plus achevée.

Spinoza y présente trois grands " genres de connaissance » $: 1$ ) la connaissance par imagination, qui est inadéquate et se subdivise en connaissance par expérience vague et en connaissance par signes (ou par oui-dire) ; 2) la connaissance par raison ou notions communes, qui est adéquate ; et 3) la science intuitive, qui est adéquate aussi et représente le moment le plus parfait du développement cognitif de l'âme.

Le premier genre de connaissance, appelé " connaissance imaginative ", " imagination » ou " opinion ", ne nous fournit pas l'idée vraie de son objet, mais seulement une idée inadéquate et tronquée. La supériorité de la raison sur ce premier mode de connaissance est donc évidente : elle fournit une idée vraie et s'accompagne de certitude. Mais Spinoza précise bien que le but de sa philosophie est de permettre de connaître le plus de choses possibles par le dernier genre de connaissance ${ }^{3}$, qui toutefois n'est pas plus «vrai » ou « adéquat »

2. À l'intérieur même de l'Éthique, l'exemple de la recherche du quatrième nombre proportionnel que donne Spinoza pour expliquer les genres de connaissance semble ne pas correspondre exactement au découpage opéré précédemment entre ceux-ci (voir E 2P40S2, G II, 122).

3. Voir par exemple le Traité de la réforme de l'entendement : "Du troisième, par contre, il faut bien dire que nous possédons là l'idée de la chose, et aussi que [par ce mode] nous faisons des inférences sans danger d'erreur. Et néanmoins, en lui-même, il n'est pas le moyen 
que le deuxième. En quoi consiste alors son privilège ? La tradition philosophique, depuis Platon et Aristote du moins, a placé la connaissance intuitive au-dessus de la connaissance rationnelle ou discursive, en opposant le caractère déductif de l'une à l'immédiateté de l'autre. Et l'on peut voir nettement chez Spinoza l'influence du modèle antique : peut-être en est-ce simplement le reflet? Mais l'explication peut aussi être ailleurs. Nous ne croyons pas que le troisième genre de connaissance dont il est question dans l'Éthique soit réellement coupé du deuxième, ce que beaucoup accorderont ${ }^{4}$, mais nous poussons cette affirmation jusqu'à proposer de voir le deuxième et le troisième genre comme une seule et même connaissance, considérée simplement dans des perspectives différentes, à des niveaux différents de son déploiement. Et plus précisément, nous émettons l'hypothèse que l'intuition est une expérience affective de puissance immédiatement liée à la connaissance par raison, dont elle n'est alors autre que la modification, de même que tout affect est la modification immédiate d'une idée. Cette conception implique naturellement qu'une « intuition » découle toujours de l'appréhension d'une chose par la raison, ce qui peut certes être discutable.

Car en quoi, dans ce cas, la supériorité de l'intuition sur la raison réside-t-elle encore ? Simplement dans le fait que ce vers quoi tend l'Éthique, c'est la joie, comme le souligne la citation placée en exergue au début de cet article. Ce n'est pas la vérité ou la connaissance en tant que telles qui constituent le bien suprême, c'est ce qui en découle : la béatitude. Mais ici la distinction est purement de raison, et c'est bien pourquoi la différence entre le deuxième et le troisième genre est purement nominale elle aussi à nos yeux. La béatitude se confond avec la connaissance de Dieu parce qu'elle en est la modification affective immédiate. L'une n'est donc rien sans l'autre : en disant qu'il faut connaitre autant que possible par le genre intuitif de connaissance, Spinoza dit seulement qu'il faut parvenir à « jouir » de la chose elle-même ${ }^{5}$, et que c'est dans cette jouissance affective que réside notre plus grand bien. Cela n'implique pas que l'on doive franchir une étape supplémentaire après être parvenu à la connaissance rationnelle d'une chose. Il n'y a qu'une " étape » : celle entre la connaissance inadéquate et la connaissance adéquate. Le reste n'est qu'une question de degré et de perspective.

d'atteindre notre perfection. Le quatrième mode seul saisit l'essence adéquate de la chose et cela sans danger d'erreur. Aussi faudra-t-il surtout user de celui-ci » (TIE 28-29, G II, 13).

4. La continuité immédiate entre les deux genres de connaissance est un fait établi dans la littérature secondaire. Par exemple elle est affirmée par Alexandre Matheron en 1969 (Individu et communauté chez Spinoza, Paris, Éditions de Minuit, 1969, en particulier p. 580582, avec l'importante note 42), et théorisée par Martial Gueroult en 1974 (Spinoza II - L'âme, op. cit., en particulier p. 467-471).

5. Songeons à l'expression du Court Traité : « Nous appelons connaissance claire celle qui s'acquiert, non par une conviction née de raisonnements, mais par sentiment de jouissance de la chose elle-même, et elle l'emporte de beaucoup sur les autres » (KV 2/2 no2, G I, 55). 
Dans tous les cas, la joie est indiscutablement présente au terme de la progression éthique. Que l'on accepte ou non l'hypothèse de lecture qui fait du troisième genre de connaissance la modification affective du deuxième, il faut convenir que le texte parle sans conteste de la joie suprême à laquelle mène la progression dans la connaissance (et que Spinoza appelle béatitude), joie en laquelle consiste le salut. Mais la joie et l'affectivité propres à la connaissance ne se trouvent pas qu'au terme du progrès, dans les formes particulièrement bien connues de l'amour envers Dieu et de l'amour intellectuel de Dieu ; elles constituent les aiguillons même du cheminement. Comment et pourquoi, sinon, apparaitraient-elles soudain ? La joie est proprement le « ressort » de la progression éthique, ce qui en explique la dynamique ascendante. Dans ce cadre, la réflexion que nous entendons mener désormais sur la place de la joie dans le passage du premier au deuxième genre de connaissance, puis dans le renforcement de la connaissance adéquate, s'avère essentielle pour comprendre l'idée même d'un «salut » humain.

\section{De l'inadéquation à l'adéquation}

Spinoza est très ferme quant à l'affirmation qu'en elle-même, aucune idée n'est fausse ${ }^{6}$ : «Toutes les idées, considérées dans leur rapport avec Dieu, sont vraies » (E 2P32, G II, 116); " Il n'y a dans les idées rien de positif à cause de quoi elles sont dites fausses » (E 2P33, G II, 116), et " La fausseté consiste dans une privation de connaissance qu'enveloppent les idées inadéquates, c'est-à-dire mutilées et confuses » (E 2P35, G II, 116). En somme, ce qui fait qu'une idée est dite fausse, c'est son inadéquation ou sa confusion, mais ces dernières ne sont que des éléments négatifs (des mutilations, des privations) et non des éléments positifs, c'est-à-dire des caractéristiques existantes de l'idée. C'est une privation d'être qui est cause de cette imperfection relative, et l'on se souviendra des belles analyses de Deleuze sur la différence entre cette privation, qui n'empêche pas une perfection de l'idée ou de la chose en elle-même (positivement), et un quelconque manque ou négation, qui serait la reconnaissance d'une imperfection en $\mathrm{soi}^{7}$. Lorsque mon âme perçoit une chose, l'idée que j'ai est celle de Dieu même, en un sens :

Quand nous disons que l'Âme humaine perçoit telle ou telle chose, nous ne disons rien d'autre sinon que Dieu, non en tant qu'il est infini, mais en tant

6. Ce point a intrigué en particulier Jonathan Bennett qui, dans « Spinoza sur l'erreur ", tente de manière plaisante de percer les raisons secrètes pour lesquelles Spinoza ne pouvait accepter l'évidence de la fausseté de certaines croyances (in Studia Spinozana, vol. 2, 1986, Walther \& Walther Verlag, p. 197-217).

7. Sur ce point, voir G. Deleuze, Spinoza - Philosophie pratique, Paris, Éditions de Minuit, 1981, en particulier p. 123-126. Voir aussi l'explication des mots de privation et de négation dans Ep. 21 à G. de Blyenberg (G IV, 128-129). 
qu'il s'explique par la nature de l'Âme humaine, ou constitue l'essence de l’Âme humaine, a telle ou telle idée (E 2P11C, G II, 94-95).

Et en Dieu, toutes les idées sont vraies. Comment alors comprendre que l'on se trompe parfois, ou qu'un progrès dans la connaissance soit nécessaire pour l'âme humaine ? L'explication réside dans le fait que ma connaissance n'est celle de Dieu qu' " en un sens ", justement ; car elle est avant tout imagination ou affection de mon corps par un objet extérieur à celui-ci :

Et quand nous disons que Dieu a telle ou telle idée, non en tant seulement qu'il constitue la nature de l'Âme humaine, mais en tant qu'il a, outre cette Âme, et conjointement à elle, l'idée d'une autre chose, alors nous disons que l'Âme humaine perçoit une chose partiellement ou inadéquatement (ibid, G II, 95).

Il faut bien comprendre que selon ce qu'indique cette citation, toute connaissance d'une chose extérieure est une connaissance partielle ou inadéquate. Il en va tout simplement ainsi parce que notre connaissance est alors celle de quelque chose d'externe à la nature de notre âme, qu'il s'agisse de notre corps ou du mode extérieur à celui-ci qui vient l'affecter. L'âme ayant une idée de cette chose extérieure "dépend " nécessairement d'elle et de la manière dont cette chose l'affecte, c'est-à-dire qu'elle est passive. Sa perception est liée à la manière particulière (et non universelle) dont la chose lui apparaît, en un temps et un lieu donnés. Mais la connaissance parfaite ou adéquate saisit la chose dans ce qu'elle a d'universel et d'éternel. Dieu, qui a conjointement à l'idée de mon corps l'idée de toutes les choses existantes, comprend l'affection que reçoit mon corps selon les lois universelles de la nature, et sa connaissance de la nécessité de cette affection dans l'ordre de la nature est une action. Tout a un sens plein ; l'affection de mon corps est replacée dans le réseau infini (E 1P28) qui l'explique et lui donne donc un sens complet. Du point de vue de Dieu, qui comprend la totalité de l'être, il n'y a ni passivité ni inadéquation. Et tout le sens de l'éthique spinoziste est précisément de nous permettre de rejoindre ce point de vue suprême.

Comment passe-t-on d'une connaissance initialement toujours inadéquate des choses extérieures à une connaissance adéquate ? La connaissance inadéquate n'est pas erronée en elle-même, de sorte qu'il s'agit simplement de la compléter par d'autres idées, qui la replacent alors dans sa vérité ${ }^{8}$. Le problème est qu'une certaine confusion peut faire que nous n'ayons pas certaines idées qui rectifieraient notre idée inadéquate pour la situer adéquatement

8. «Ici, pour commencer d'indiquer ce qu'est l'erreur, je voudrais faire observer que les imaginations de l'Âme considérées en elles-mêmes ne contiennent aucune erreur ; autrement dit, que l'Âme n'est pas dans l'erreur, parce qu'elle imagine ; mais qu'elle est dans l'erreur, en tant qu'elle est privée d'une idée qui exclut l'existence de ces choses qu'elle imagine comme lui étant présentes. Si en effet l'Âme, durant qu'elle imagine comme lui étant présentes des choses n'existant pas, savait en même temps que ces choses n'existent pas en réalité, elle attribuerait certes cette puissance d'imaginer à une vertu de sa nature, non à un vice » (E 2P17S, G II, 106. C'est nous qui soulignons). 
dans l'ensemble de la nature. Spinoza prend lui-même, à plusieurs reprises, un exemple fort éloquent à ce sujet. Lorsque nous voyons le soleil, nous imaginons un astre peu éloigné de nous. En elle-même, toutefois, notre perception du soleil n'est pas trompeuse : c'est seulement l'absence d'une idée de la taille ou de la distance réelles du soleil qui nous font l'imaginer proche. Notre perception ou notre imagination changent-elles lorsque cette idée supplémentaire nous est donnée ? Aucunement, puisqu'elle est, en elle-même, vraie, et qu'elle ne fait qu'exprimer la manière dont notre corps est réellement affecté par le soleil. Cette vérité de l'affection (affectio) de mon corps, c'est-à-dire de l'état dans lequel mon corps est par rapport à une chose extérieure qui l'affecte, est simplement enrichie par une autre idée qui permet de " redresser » l'interprétation que je donne de cette sensation ou perception première :

Plus tard, en effet, tout en sachant que le soleil est distant de plus de 600 fois le diamètre terrestre, nous ne laisserons pas néanmoins d'imaginer qu'il est près de nous ; car nous n'imaginons pas le soleil aussi proche parce que nous ignorons sa vraie distance, mais parce qu'une affection de notre Corps enveloppe l'essence du soleil, en tant que le Corps lui-même est affecté par cet astre (E 2P35S, G II, 117).

Dans ce cas, la perception qu'on a par l'imagination seule est enrichie d'une autre idée à laquelle elle est comparée et à l'aune de laquelle elle prend un sens différent, et l'on connaît adéquatement, c'est-à-dire que l'idée est en nous comme elle est en Dieu : vraie. L'idée de comparaison est ici essentielle, et c'est elle qui permet d'expliquer la formation de notions communes, c'està-dire d'une connaissance rationnelle.

Dans une analyse fine et précise des processus cognitifs chez Spinoza, Pierre-François Moreau parle certes d'une «rupture » entre le premier et le deuxième genre de connaissance, mais il maintient fermement la possibilité du passage de l'un à l'autre d'une manière particulièrement audacieuse, en parlant de types d'imagination à l'œuvre dans les deux genres ${ }^{9}$. Cette interprétation originale mérite d'être bien comprise. Il y a, propose-t-il, trois façons différentes d'imaginer, dont on comprend que la troisième, fort différente des deux premières, est déjà presque la raison, à laquelle correspond le deuxième genre de connaissance : il y a l'imagination vive, qui " procède par association [et] donne l'ingenium du prophète " (premier type de connaissance dans le premier genre) ; il y a l'imagination pratique, qui «procède par répétition [et] donne l'empirique » (deuxième type de connaissance dans le premier genre) ; et il y a l'imagination puissante, qui «favorise la comparaison [et] donne l'homme d'entendement ${ }^{10}$. Ce troisième type d'imagination d'après Moreau, l'imagination comparative, serait ce qui me permet de comparer ma perception par expérience vague (ou par ouï-dire) avec les autres choses et d'y adjoindre une

9. P.-F. Moreau, Spinoza. L'expérience et l'éternité, Paris, PUF, 1994, p. 254-256.

10. Ibid., p. 255. 
connaissance venant rectifier l'interprétation fautive que j'en donnais en l'absence de cette comparaison. La raison est donc, si l'on veut bien suivre l'indication donnée par Moreau et même reformuler son hypothèse de manière plus tranchée encore, une forme d'imagination. Sans nécessairement demander l'adhésion à ce découpage en types d'imagination, nous croyons que cette interprétation vise juste en traçant un pont entre imagination et raison, sans nier l'irréconciliabilité des deux premiers types d'imagination avec la connaissance vraie, et surtout, en soulignant le rôle primordial de la comparaison. C'est exactement ainsi que se forment les " notions communes " propres à l'Éthique (cf. E 2P38-39 et leurs corollaires). Le passage au deuxième genre de connaissance, qui correspond à la découverte de l'idée vraie, se fait donc sans violence par rapport au premier genre de connaissance. Pourtant, c'est ce passage qui représente l'étape la plus importante dans le développement de la connaissance.

Si l'on comprend bien, dès lors, comment le passage du premier au deuxième genre de connaissance se fait en théorie, c'est-à-dire par l'accession à une compréhension de l'universalité des choses grâce à la comparaison entre elles des perceptions - ce qu'aucun animal ne saurait d'ailleurs faire, selon Spinoza - , on ne voit pas pour autant encore ce qui justifierait que l'âme, après avoir eu une idée commune, cherche à connaître toute chose de manière adéquate. Spinoza est ainsi confronté à ce qui représente certainement le problème fondamental de toute éthique : en quoi le caractère supérieur du bien qu'elle propose réside-t-il ? En d'autres termes, pourquoi faudrait-il réformer son mode de vie pour se conformer à ce modèle ? et, de manière plus problématique encore, comment cette sortie d'une vie non éthique vers une vie éthique est-elle possible ? C'est toute la question du Traité de la réforme de l'entendement. Mais c'est plus spécifiquement la théorie des affects passifs et actifs qui joue dans le système spinoziste le rôle de déclencheur de la dynamique du progrès. Car seul un élément dynamique peut vaincre l'inertie de l'acrasie. Et Spinoza, plus que tout autre, est conscient de l'insuffisance des mots sans le vécu, de la théorie sans l'expérience intérieure : "La connaissance vraie du bon et du mauvais ne peut, en tant que vraie, réduire aucun affect, mais seulement en tant qu'elle est considérée comme un affect » (E 4P14, G II, 219). En d'autres termes, contre les affects (affectus, c'est-à-dire les sentiments, les émotions) qui nous sont néfastes ou expriment un état diminué de notre être, et qu'avec Spinoza on peut appeler les passions tristes, seuls des affects peuvent agir. Et seulement des affects exprimant la joie ou le désir, c'està-dire posant une plus grande puissance de notre être, et de surcroît réellement très puissants ou en grand nombre, car les passions, elles, sont nombreuses et peuvent même écraser un affect individuellement plus puissant : "Un Désir qui naît de la connaissance vraie du bon et du mauvais, peut être éteint ou réduit par beaucoup d'autres Désirs naissant des affects par lesquels nous sommes dominés » (E 4P15, G II, 220). Mais malgré la difficulté que traduit bien cette citation, c'est bien dans la lutte des affects les uns contre les autres, secondée par la connaissance, que réside la possibilité de faire son salut. 
La troisième partie de l'Éthique décrit le fonctionnement du domaine affectif et tente d'expliquer la totalité de nos comportements en termes de mécanisme causal strict. La théorie de la passivité et de l'activité de l'âme qui y est fournie s'avère fondamentale pour comprendre la possibilité de se libérer de l'enchaînement passionnel ${ }^{11}$ : " Notre âme est active en certaines choses, passive en d'autres, savoir, en tant qu'elle a des idées adéquates, elle est nécessairement active en certaines choses; en tant qu'elle a des idées inadéquates, elle est nécessairement passive en certaines choses » (E 3P1, G II, 140) ; " Il suit de là que l'Âme est soumise à d'autant plus de passions qu'elle a plus d'idées inadéquates, et, au contraire, est active d'autant plus qu'elle a plus d'idées adéquates " (E 3P1C, G II, 141). En d'autres termes, si une personne passe d'une idée inadéquate d'une chose à une idée adéquate, elle devient ellemême la cause de son idée et sa puissance d'agir s'en trouve augmentée (selon E 3P1 et son corollaire). Autrement dit, son essence formelle, qui correspond à un certain degré de puissance exprimée, progresse vers une plus grande perfection. Or c'est là précisément la définition de la joie (laetitia) : « La joie est le passage de l'homme d'une moindre à une plus grande perfection » (E 3DA2, G II, 191), ce qui revient à dire que tout progrès cognitif est immédiatement joyeux. Inversement, toute perte de puissance d'une personne s'accompagne de tristesse (tristitia), qui n'est rien d'autre que « le passage de l'homme d'une plus grande à une moindre perfection » (E 3DA3, G II, 191). Et joie et tristesse, avec le désir (cupiditas) qui n'est autre que l'expression consciente première de l'essence formelle de la chose, de son conatus, sont les affects fondamentaux (E 3P11S, G II, 149), ce qui signifie qu'on peut en faire dériver toute la gamme des affects jusqu'aux plus complexes, comme l'effectue précisément Spinoza dans la troisième partie de l'Éthique.

La passivité et l'activité ne recoupent ainsi pas exactement la joie et la tristesse, et alors que toute activité est joyeuse (elle correspond à une connaissance adéquate), une passion peut être joyeuse ou triste, selon la manière dont l'individu est affecté vers plus de vérité ou moins au sein d'une connaissance de la chose extérieure qui reste tout de même inadéquate. Si l'on passe d'une connaissance très inadéquate de la chose à une connaissance un peu moins inadéquate mais toujours partielle, on " progresse " bel et bien (donc on éprouve de la joie), mais on reste passif. Si en revanche on passe d'une connaissance inadéquate à une connaissance adéquate de la chose, cette joie trouve sa cause en nous puisque la connaissance s'accompagne d'une conscience de la puissance de penser qu'on exprime, et la joie est active. L'activité et la passivité ont donc à voir avec le fait qu'on soit ou non la cause adéquate de notre connaissance, tandis que la joie et la tristesse dépendent de la tran-

11. Pour un approfondissement de la notion de passivité, cf. Pascal Séverac, "Passivité et désir d'activité chez Spinoza ", in Spinoza et les affects, dir. F. Brugère et P.-F. Moreau, Travaux et documents du groupe de recherches spinoziste, $\mathrm{n}^{\circ} 7$, Paris, Presses de l'Université Paris-Sorbonne, 1998, p. 39-54. 
sition d'un état de puissance à un autre — sachant que l'on est d'autant plus puissant que l'on connaît adéquatement, c'est-à-dire que la part de fausseté inhérente à toute idée de chose extérieure est réduite.

Il nous paraît clair que c'est par cet ancrage dans l'expérience affective de la joie que la théorie éthique de Spinoza permet de justifier la naissance d'un nouveau désir, qui est à la base de la démarche du progrès individuel : le désir de connaître. Ce désir de se servir de son entendement, de connaître les choses de plus en plus par sa raison, et de moins en moins par l'imagination seule, ne peut, en tant qu'affect, être engendré que par un autre affect (E 4P7, G II, 214). Et c'est ainsi que la joie initiale qui accompagne la formation d'une première idée vraie reste dans l'esprit comme une marque indélébile, capable de lui servir à tout instant de critère du vrai et du faux — nous songeons au passage célèbre qui présente l'idée vraie, et la certitude qui la caractérise, comme "norma sui, et falsi », norme d'elle-même et du faux (E 2P43S, G II, 124). Sans doute une seule vraie idée ne suffit-elle pas : nous avons tous d'ailleurs au moins une idée vraie, au moins une notion commune qui nous révèle la nature divine ${ }^{12}$, et pourtant cela ne suffit pas à faire de nous des sages. Mais on peut supposer qu'avec la répétition d'une telle expérience, ainsi qu'avec la pratique de la logique qui entraîne l'esprit à penser rigoureusement ${ }^{13}$, une habitude du vrai peut s'installer et que l'âme, dès lors, peut réellement s'auto-perfectionner.

L'idée d'un auto-perfectionnement de l'âme est essentielle pour que le salut soit possible. Spinoza prend, dans le Traité de la réforme de l'entendement, l'image éloquente d'un outil simple qui sert à en faire un plus perfectionné, et ce dernier à son tour un plus perfectionné, et ainsi de suite, comme l'entendement qui à partir d'une idée vraie peut construire d'autres idées vraies et progresser seul vers la connaissance parfaite (TIE 85, G II, 32) ${ }^{14}$. Notre âme n'est ainsi guère plus qu' "une espèce d'automate spirituel " (idem), répondant nécessairement par un certain sentiment à un certain autre ou à une certaine situation. Or c'est parce que la joie qui accompagne la formation d'idées adéquates donne envie d'en rechercher d'autres, et surtout, de ne plus se contenter des connaissances inadéquates, que l'entendement sait se former et se rectifier. C'est en cela précisément que l'entendement peut, dès

12. «L'âme humaine a une connaissance adéquate de l'essence éternelle et infinie de Dieu » (E 2P47, G II, 128).

13. C'est à la logique qu'est dévolu le rôle d'habituer notre entendement au vrai, d'augmenter son discernement et sa puissance : "Quant à la manière de porter l'Entendement à sa perfection et à la voie y conduisant, ce sont choses qui n'appartiennent pas au présent ouvrage, non plus que l'art de traiter le Corps de façon qu'il puisse remplir convenablement sa fonction ; cette dernière question est du ressort de la Médecine, l'autre de la Logique » (E 5Praef, G II, 277). C'est comme si la logique était vue comme un exercice préliminaire à la lecture de l'Éthique.

14. Voir également Wim N. A. Klever, qui a développé une belle interprétation du caractère automatique de la connaissance dans "Quasi aliquod automa spirituale ", Proceedings of the First International Congress on Spinoza: Spinoza nel 350 Anniversario della nascita, Urbino 4-8 ottobre 1982, dir. Emilia Giancotti, Naples, Bibliopolis, 1985, p. 249-257. 
sa première idée adéquate, se perfectionner seul, trouvant en soi le critère de sa rectitude tout autant que le moteur de sa dynamique progressive. Ce moteur, c'est donc l'affect de joie, qui est d'autant plus grand que la connaissance est plus active. Par cette expérience d'accroissement de puissance, l'entendement se renforce de plus en plus et finit par se guider seul vers une connaissance plus parfaite. Il agit alors vraiment comme un automate spirituel, qui, seul et par ses seules lois, tend à l'éternité et à la béatitude. L'homme est cependant entravé par l'ignorance des vraies causes des choses qui détournent le désir de son véritable objet et le rendent sujet aux passions. Mais ce perfectionnement de la connaissance n'en est pas moins naturellement inscrit dans l'essence puissante de l'entendement, et c'est pourquoi il faut le libérer.

\section{Vers un approfondissement indéfini de la connaissance vraie}

Nous espérons être parvenue à faire voir par les quelques explications qui précèdent quel rôle revient certainement aux affects dans le passage du premier genre de connaissance au deuxième, et plus particulièrement à la joie, pour expliquer la possibilité de développer un désir réel de connaître adéquatement, ou de ne plus se contenter de la connaissance imaginative, qui laisse passif. Cette étape fondamentale supposée passée, on peut se demander à quoi correspond la nouvelle distinction opérée par Spinoza entre d'autres « genres de connaissance ", car cette fois c'est au sein de la connaissance adéquate qu'elle s'opère. Mais une idée n'est aucunement plus complète ou plus adéquate dans le troisième genre de connaissance que dans le deuxième, puisque dès le deuxième elle est déjà parfaitement adéquate en l'âme humaine. Qu'estce qui, dans ce cas, diffère dans la "science intuitive " et explique la distinction entre deuxième et troisième genre de connaissance ?

Ce qui explique ce passage c'est, une fois encore, une différence dans le mode de perception de cette idée ou perception, qui en elle-même (c'est-à-dire en Dieu) est toujours la même, unique et éternelle, à travers les trois genres de connaissance. Le troisième genre, explique Spinoza, "procède de l'idée adéquate de l'essence formelle de certains attributs de Dieu à la connaissance adéquate de l'essence de ces choses » (E 2P40S2, G II, 122). Qu'entend-il par " essence formelle de certains attributs de Dieu ", et d'où provient la connaissance qu'on en a ? C'est nécessairement dans le deuxième genre que nous acquérons cette connaissance, sans quoi il y aurait un hiatus infranchissable entre le deuxième et le troisième genre.

Notre idée formée selon le deuxième genre, avons-nous dit, est identique en Dieu et en nous, c'est-à-dire est vraie. C'est là la source de son redoublement sous la forme d'une conscience de soi que Spinoza énonce en $\mathrm{E}$ 2P43 : "Qui a une idée vraie sait en même temps qu'il a une idée vraie et ne peut douter de la vérité de sa connaissance » (G II, 123). Cette idée avait déjà été introduite en E 2P20, en E 2P21 et E 2P21S : 
L'existence de l'idée de l'Âme, dis-je, et celle de l'Âme elle-même suivent en Dieu avec la même nécessité de la même puissance de penser. Car, en réalité, l'idée de l'Âme, c'est-à-dire l'idée de l'idée, n'est rien d'autre que la forme de l'idée, en tant que celle-ci est considérée comme un mode du penser sans relation avec l'objet ; de même quelqu'un qui sait quelque chose sait, par cela même, qu'il le sait, et il sait en même temps qu'il sait qu'il sait, et ainsi à l'infini. Mais de cela il sera question plus tard (E 2P21S, G II, 109).

En d'autres termes, comme il le développe effectivement "plus tard » en $\mathrm{E} 2 \mathrm{P} 43 \mathrm{D}$ et $\mathrm{S}$, la possession d'une idée vraie ou adéquate enveloppe une certitude qui est celle de la possession de la vérité, et qui prend la figure d'une conscience immédiate et puissante d'être dans le vrai lorsqu'on a une idée adéquate. L'âme qui comprend quelque chose a simultanément conscience de comprendre cette chose et de ne pas se tromper, d'où l'idée déjà citée qu'elle se suffit à elle-même pour s'éclairer. La même notion de certitude se dégage du paragraphe 35 du Traité de la réforme de l'entendement:

La certitude n'est rien d'autre que l'essence objective elle-même ; c'est-à-dire, que la manière, dont nous sentons l'essence formelle, est la certitude elle-même. D'où il apert à nouveau que pour avoir la certitude de la vérité il n'est besoin d'aucun autre signe que la possession de l'idée vraie. En effet, ainsi que nous l'avons montré, point n'est besoin, pour que je sache, de savoir que je sais ${ }^{15}$.

Il nous semble que ces éléments pointent vers une certaine interprétation du troisième genre de connaissance, de la "science intuitive ». Nous voudrions tout simplement proposer de considérer que la perception de la " forme de l'idée » qui est donnée à l'âme lorsqu'elle connaît de manière adéquate, c'est-à-dire de l'essence formelle de cette idée ou de son intensité propre dans l'ordre de la puissance du penser, est une perception de la forme de l'attribut pensée lui-même, c'est-à-dire du type de puissance qui est exprimé par la pensée. Ce point est également rendu intelligible par l'emploi de "nous sentons " dans la citation ci-dessus pour caractériser la connaissance de l'essence formelle : cette sensation est un sentiment, un affect, c'est-à-dire une expérience de puissance. Et de quelle puissance ? De même que l'essence formelle d'un corps est le rapport unique de mouvement et de repos qui unit ses parties dans un même conatus ${ }^{16}$ — l'essence du corps est ce conatus luimême - l'essence formelle de l'âme n'est rien d'autre que le degré précis de puissance divine qu'elle exprime, soit de nouveau ce conatus, tel qu'il s'exprime dans la pensée. Et il en va de même pour toute idée, modification de l'essence de Dieu en tant que " chose pensante »(E 2P1), c'est-à-dire en tant que modification de l'attribut pensée ${ }^{17}$. Autrement dit, il est donné à

15. TIE 35, G II, 15. C'est nous qui soulignons.

16. Voir la définition de l'Individu dans l'exposé de la théorie des corps dans la deuxième partie de l'Éthique (E 2P13Def, G II, 99-100).

17. C'est d'ailleurs ce que signifie l'affirmation par Spinoza que « les essences formelles des choses singulières, ou modes, sont contenues dans les attributs de Dieu » (E 2 P8, G II, 90). 
l'âme à chaque perception vraie une idée de sa propre "essence formelle " qui n'est autre qu'une idée du degré précis de puissance qu'elle exprime.

Nous irions plus loin en disant que toute idée conçue selon le deuxième genre de connaissance l'est aussi par le troisième de manière immédiate, c'est-à-dire que le troisième genre de connaissance découle du deuxième comme l'affect découle de l'idée. Car selon les citations données ci-dessus sur le redoublement immédiat de l'idée vraie en idée d'idée, c'est-à-dire en conscience de soi ou essence objective ou certitude, toute idée vraie perçue par notre âme cause immédiatement en celle-ci une conscience d'elle-même et de la vérité de son penser. Il nous semble alors possible, et fructueux, de considérer que le troisième genre de connaissance correspond à l'expérience affective du deuxième, est une manière d'en éprouver la puissance. Dans ce cadre toutefois, il faut reconnaître qu'il est impossible de concevoir une idée adéquate qui ne serait pas également perçue selon le troisième genre de connaissance, ce que tous n'accorderont pas. Tentons pourtant d'en approfondir l'hypothèse.

À l'aune de cette piste d'interprétation, on peut relire la description du troisième genre de connaissance comme " [procédant] de l'idée adéquate de l'essence formelle de certains attributs de Dieu à la connaissance adéquate de l'essence de ces choses " (E 2P40S2, G II, 122) en l'expliquant de la manière suivante : dans le deuxième genre de connaissance, chaque idée enveloppe une connaissance de la puissance divine qu'elle exprime, de la force du " penser vrai ». Si l'on part de cette connaissance ou conscience, on peut alors connaître l'essence formelle des corps qui sont les objets de nos idées, et en même temps le type de puissance exprimé par notre propre esprit. Il faut alors comprendre l' ' intuition » dont il est question dans le troisième genre de connaissance comme une connaissance expérientielle, une expérience de la puissance divine, et voir en celle-ci une connaissance des essences formelles des choses et des attributs de Dieu au sens où nous l'avons expliqué précédemment, c'est-à-dire des degrés propres de puissance. Ainsi, la connaissance adéquate correspondrait à une conscience de la puissance infinie divine qui est cause de toutes choses, et ce sous deux aspects : l'attribut étendue (par la compréhension par notions communes d'un corps), et l'attribut pensée (par la découverte de la certitude qui exprime la puissance infinie de penser divine) ; et à une expérience du degré de puissance précisément exprimé par soi-même et l'objet connu.

C'est cette expérience de la puissance divine qui explique le fameux "sentimus, experimurque, nos aeternos esse ", "nous sentons, et nous savons par expérience que nous sommes éternels » de E 5P23S (G II, 196); et toute la cinquième partie de l'Éthique se donne à lire de manière particulièrement significative si l'on accepte de voir dans l'intuition une connaissance expérientielle, dont la vivacité est celle d'un partage de puissance, d'une compréhension vécue, intérieure, de la puissance de l'être qu'on exprime et qu'exprime toute chose. C'est en ce sens que " nous sentons » notre éternité, mais il va de soi que 
ce n'est pas une sensation du type de celles que nous éprouvons dans le premier genre de connaissance lorsque nous subissons une affection. Plutôt que la sensation de l'affection (affectio) corporelle, c'est la sensation de l'affect (affec$t u s)$, c'est le sentiment, l'émotion qui accompagne toute idée, et c'est ici une perception adéquate ${ }^{18}$.

Ce qu'il nous importe de souligner étant avant tout le rôle des affects, notons que la certitude peut se voir attribuer un rôle de moteur pour glisser du deuxième genre de connaissance au troisième. Le deuxième genre est alors compris comme la perception d'un corps sous l'angle de l'éternité ou par notion communes, et le troisième comme l'expérience même de la puissance infinie divine (car le troisième genre de connaissance procède de la « connaissance de l'essence formelle de certains attributs de Dieu » : l'étendue et la pensée) et du degré précis d'intensité ontologique que possède, dans cette puissance infinie, l'objet compris ( « à la connaissance adéquate de l'essence de ces choses »). L'un et l'autre ne sont alors que les deux faces, l'une cognitive et l'autre expérientielle, du même acte d'intellection, de saisie de l'essence formelle d'une chose singulière. La certitude, qui en tant qu'affect actif (puisqu'elle provient de la connaissance vraie) est nécessairement dérivée du désir ou de la joie (E 3P59, G II, 188), explique d'où provient le désir d'approfondissement sans fin de la connaissance adéquate par l'âme. Ainsi, le deuxième et le troisième genre de connaissance fusionnent dans un cercle d'interaction sans fin. La lecture faisant de l'intuition un genre " affectif » de connaissance, une perception affective de la forme des attributs de la pensée et de l'étendue, et de la place précise qu'occupe l'essence de la chose dans cette puissance infinie, permet donc de justifier que l'âme ne s'en tienne pas à une seule connaissance vraie mais, par la joie éprouvée, désire ardemment connầtre de plus en plus de choses par ce même genre de connaissance, et en ait de plus en plus le pouvoir. Car la joie éprouvée est un renforcement dans sa perfection, ou sa puissance.

Enfin, l'affectivité joyeuse ne se trouve pas seulement à la source du passage d'un genre de connaissance à l'autre, mais elle en est aussi le résultat, ce qui explique que, de manière parfaitement compréhensible, différentes formes d'amour correspondent à la connaissance adéquate : l'amour envers Dieu (amor erga Deum), introduit en E 5P15 ${ }^{19}$, l'amour intellectuel de Dieu

18. Étant donné la portée et le but de cet article, nous n'entendons pas discuter ici les deux interprétations les plus importantes et les plus récentes du sentiment d'éternité, celle de Pierre-François Moreau (op. cit.) et de Chantal Jaquet (Sub specie ceternitatis. Étude des concepts de temps, durée et éternité chez Spinoza, Paris, Kimé, 1997). Notons simplement que nous sommes plus proche de la deuxième lecture que de la première, qui affirme notamment que ce sentiment est relié à une connaissance imparfaite.

19. "Qui se connaît lui-même, et connaît ses affections clairement et distinctement, aime Dieu et d'autant plus qu'il se connaît plus et qu'il connaît plus ses affections » (E 5P15, G II, 290). Cet amour découle du rattachement de toutes les choses finies existantes, en tant qu'elles sont conçues sub duratione, à l'idée de Dieu comme cause. 
(amor Dei intellectualis), caractérisé en E 5P3220, et finalement l'amour intellectuel de l'âme envers Dieu (Mentis amor intellectualis erga Deum) décrit en E 5P36 ${ }^{21}$. Une fois encore, un approfondissement de ces types d'amour irait au-delà de l'objectif que nous nous sommes proposé ici, et nous devons l'écarter. Nous nous bornerons pour notre propos actuel à souligner la présence réelle de ces formes joyeuses de l'affectivité et leur importance évidente aux yeux de Spinoza pour comprendre ce qu'est le salut. Le salut, c'est précisément la joie infinie découlant de la connaissance de l'infini ; ou encore, pour le dire autrement, la béatitude n'est autre que la connaissance de la nécessité divine, en tant que cette connaissance s'accompagne d'une joie infiniment puissante. La béatitude est ainsi la connaissance selon le troisième genre elle-mêmes - ce qui explique que l'âme humaine tende indéfiniment vers elle. Et la béatitude se fait amour réciproque de l'âme et de Dieu :

Nous connaissons clairement par là en quoi notre salut, c'est-à-dire notre Béatitude ou notre Liberté, consiste ; je veux dire dans un Amour constant et éternel envers Dieu, ou dans l'Amour de Dieu envers les hommes (E 5P36S, G II, 303).

L'amour, en effet, se définit comme "une joie qu'accompagne l'idée d'une chose extérieure " (E 3P13S). Or, qu'est-ce connaître selon le troisième genre de connaissance ? C'est, tout d'abord, connaître de manière adéquate, et donc éprouver de la joie, étant donné que l'on passe d'une connaissance inadéquate de la chose - connaissance selon le premier genre - à une connaissance adéquate - selon le deuxième genre. Connaître selon le troisième genre, pour le dire autrement, c'est connaître à la fois selon le premier et selon le deuxième genre, et ressentir la joie du rétablissement dans la vérité de l'idée donnée confusément à la base. Deuxièmement, selon ce que nous avons développé précédemment, c'est éprouver la puissance infinie divine qui s'exprime dans le " penser vrai » et c'est donc savoir que l'on sait, autrement dit se connaître soi-même comme cause adéquate en tant que l'on est puissant. Or la puissance est l'essence même de Dieu (E 1P34), de sorte qu'il est certain qu'en éprouvant la puissance divine, comme c'est le cas si on con-

20. «À tout ce que nous connaissons par le troisième genre de connaissance nous prenons plaisir, et cela avec l'accompagnement comme cause de l'idée de Dieu » (E 5P32, G II, 300). Dieu est ici aimé en tant que cause éternelle des choses, conçues elles aussi sub specie ceternitatis. La distinction que nous traçons ici entre l'amour envers Dieu et l'amour intellectuel de Dieu s'inspire de la distinction faite par Chantal Jaquet entre connaissance sub quadam specie aternitatis et connaissance sub specie aternitatis (voir Ch. Jaquet, op. cit, p. 118-120).

21. "L'Amour intellectuel de l'Âme envers Dieu est l'amour même duquel Dieu s'aime lui-même, non en tant qu'il est infini, mais en tant qu'il peut s'expliquer par l'essence de l'Âme humaine considérée comme ayant une sorte d'éternité ; c'est-à-dire l'Amour intellectuel de l'Âme envers Dieu est une partie de l'Amour intellectuel infini duquel Dieu s'aime lui-même " (E 5P36, G II, 302). Pour comprendre cette réciprocité et même cette identité des deux amours (voir E 5P36C), on se référera aux analyses incontournables d'Alexandre Matheron (op. cit., p. 598-599). 
naît adéquatement, on connaît l'essence formelle de Dieu. Connaître selon le troisième genre de connaissance, c'est donc éprouver de la joie, ce qui était le premier point, et rattacher cette joie éprouvée à l'idée de Dieu, ce qui était le deuxième : c'est donc éprouver de l'amour envers Dieu. L'âme humaine retrouve ainsi sa place adéquate dans l'entendement divin infini en se confondant pleinement avec l'idée parfaite que Dieu a de notre corps sous l'aspect de l'éternité, et elle participe de la jouissance infinie et universelle de Dieu contemplant activement son auto-production. Le salut est, on le voit bien, une joie infinie.

Ainsi espérons-nous avoir montré l'importance d'une prise en considération des affects, et de la joie en particulier, pour rendre compte de la progression éthique en général, laquelle ne se réduit assurément pas à une progression dans la " connaissance » si l'on entend par celle-ci un simple contenu cognitif, théorique. La vie affective n'est pas un ornement ou un vernis posé sur la vie cognitive, mais la vie même qui l'anime, le souffle qui la fait progresser, et ce qui nous permet de l'éprouver. Or les affects sont donnés dans les trois genres de connaissance, et dans le deuxième genre, ils prennent la forme de la conscience de soi qui entraîne immédiatement la conscience de la puissance de former des idées qu'on exprime. Pour peu qu'on laisse notre entendement progresser selon les outils qu'il possède, la connaissance devient naturellement - automatiquement — joie et amour. Le salut et la béatitude résident alors simplement dans une connaissance unifiée de notre être et de toutes choses, connaissance donnée dans une expérience affective de la puissance infinie de l'être divin. Spinoza est donc tout aussi éloigné d'un rationalisme exsangue que d'un mysticisme illuminé : c'est la connaissance qui mène à Dieu, et cette connaissance est une expérience joyeuse et vive ${ }^{22}$.

22. Ce texte s'appuie sur l'interprétation développée dans ma thèse de doctorat réalisée de 1996 à 2001 à l'Université d'Ottawa, et ses idées principales ont été présentées au congrès annuel de l'Association canadienne de philosophie à l'Université Laval en mai 2001. Je tiens à remercier le CRSH (bourse de doctorat et bourse William E. Taylor) et le gouvernement de l'Ontario (bourse BÉSO) pour leur soutien dans la réalisation de mes recherches doctorales, ainsi que l'Université d'Ottawa, et en particulier son Fonds de Développement Académique, pour les nombreuses bourses et subventions de voyages accordées durant cette période. 